\title{
UM MODELO DE CÁLCULO DE TRAJETÓRIA ÓTIMA PARA MINIMIZAÇÃO DO TEMPO DE VOLTA APLICADO A UMA MOTOCICLETA DE COMPETIÇÃO
}

\author{
Rodrigo Lacerda Villalobos ${ }^{1}$ e Marcelo Massarani ${ }^{1}$ \\ ${ }^{1}$ Escola Politécnica da Universidade de São Paulo \\ E-mails: rodrigo.villalobos@gmail.com, massara@usp.br;
}

\begin{abstract}
RESUMO
O mercado de motocicletas de alta cilindrada esteve em alta no Brasil durante a década de 2010, nesse período, o número de interessados em desenvolver suas habilidades como piloto acompanhou a alta nas vendas de novas motocicletas. $\mathrm{O}$ aumento do interesse por motocicletas esportivas também aqueceu as competições, e "trackdays", onde pilotos iniciantes levam bastante tempo até adquirir as habilidades necessárias para ter tempos competitivos. Nesse ambiente, o desenvolvimento de um algoritmo que apoie pilotos e equipes de competição, indicando a trajetória ótima que uma motocicleta deve percorrer em pista, bem como os locais de aceleração e frenagem parece interessante. Tal algoritmo torna-se ainda mais atrativo se fornecer os parâmetros básicos de regulagem da motocicleta, reduzindo assim o tempo de aprendizado dos pilotos e, simultaneamente, fornecendo parâmetros teóricos de ajuste da motocicleta para os engenheiros de pista.
\end{abstract}

\section{INTRODUÇÃO}

O mercado de motocicletas esportivas de alta cilindrada no Brasil tem se mantido bastante promissor na década de 2010, onde grandes fabricantes têm investido no país, não apenas importando veículos, mas fabricando alguns modelos localmente. Motocicletas de aproximados $200 \mathrm{cv}$ de potência estão bastante presentes nas ruas, nas competições amadoras e "trackdays", onde pilotos com pouca experiência em pilotagem esportiva levam bastante tempo até adquirir a habilidade de pilotagem necessária para ter tempos competitivos. 
Nesse cenário de mercado, desenvolver equipamentos e metodologias que auxiliem esses pilotos no aprendizado torna-se interessante tanto pelo objetivo econômico, ao atender um crescente mercado consumidor de alta renda, quanto à clara questão de segurança. Assim, há espaço para desenvolvimento de um algoritmo que possa calcular para o piloto a trajetória mais eficiente, o nível de inclinação que se pode atingir, bem como os pontos ideais de aceleração e frenagem.

Em paralelo, com o crescimento do consumo de motocicletas de alta cilindrada, impulsionam-se os campeonatos locais, e torna-se também necessário desenvolver metodologias que auxiliem aos engenheiros de pista a determinar rapidamente quais parâmetros da motocicleta devem ser alterados para que se tenha uma condução facilitada, eficiente e segura.

\section{Modelo de motocicleta}

O modelo dinâmico implementado neste trabalho foi desenvolvido em [1] e foi escolhido por representar uma favorável relação entre complexidade de implantação, quantidade de parâmetros necessários e abrangência da resposta. Para definição da trajetória ótima, será utilizado o controle ótimo do processo, de forma similar ao visto em [2].

\subsection{Geometria da motocicleta}

A motocicleta pode ser descrita geometricamente pelos seguintes parâmetros:

- O ângulo de inclinação da balança de suspensão traseira em relação ao plano horizontal, representado por $\beta$;

- O ângulo de esterço, ou "steering angle" em inglês, representado por $\delta$;

- A inclinação do garfo de suspensão dianteira, ou "caster angle" em inglês, representado por $\gamma$;

- O ângulo de rotação em relação ao eixo vertical, ou "yaw angle" em inglês, representado por $\psi$;

- O ângulo de inclinação em relação ao plano vertical, representado por $\varphi$;

- Distância entre eixos, ou "wheelbase" em inglês, representada por $\omega$;

- As letras $x$ e y representam o sistema de coordenadas na motocicleta, onde $x$ refere-se ao eixo longitudinal e y ao eixo transversal.

Todos estes parâmetros podem ser observados nas figuras 1 e 2 .

\subsection{Cinemática}

O modelo da cinemática da motocicleta implementado segue o desenvolvimento descrito em [1]. 


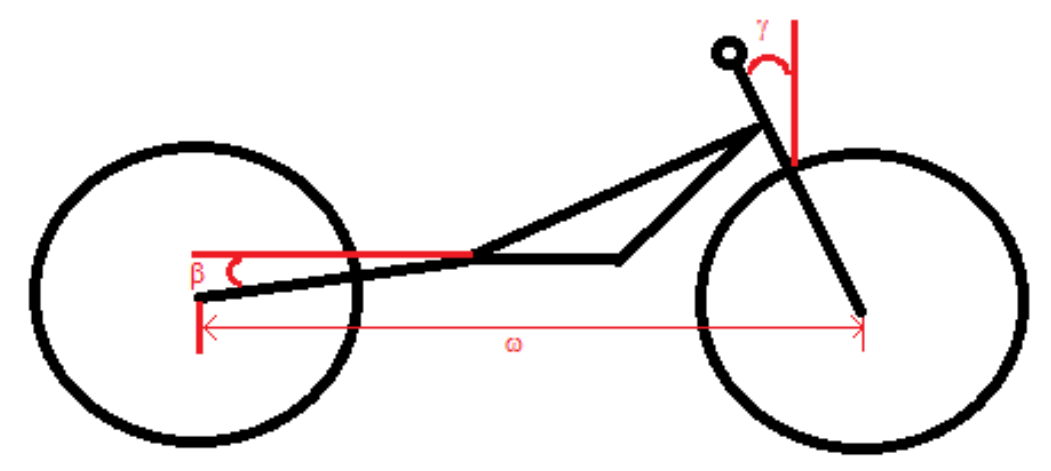

Figura 1 - Geometria da motocicleta (visão lateral)

Assim, as equações que definem a cinemática da motocicleta são:

$$
\begin{gathered}
\dot{x}=v \cos \psi \\
\dot{y}=v \sin \psi \\
\dot{\psi}=\frac{v \tan (\delta \cos \gamma)}{\omega \cos \varphi}
\end{gathered}
$$

Considerando-se que o comprimento da suspensão dianteira $l$ e o ângulo da suspensão traseira $\beta$ variam de acordo com a movimentação do centro de massa, tem-se:

$$
\begin{gathered}
\gamma=\tan ^{-1}\left(\frac{l}{t}\right)-\sin ^{-1}\left(\frac{s}{t} \sin \beta \cos \left(\tan ^{-1}\left(\frac{l}{t}\right)\right)\right) \\
\omega=s \cos \beta+t \cos \gamma+l \sin \gamma
\end{gathered}
$$

Onde t é a distância entre o centro de rotação da balança traseira e o garfo dianteiro, e s é a distância entre o centro de rotação da balança traseira e o centro da roda traseira.

\subsection{Modelo de suspensão}


O modelo aqui descrito tem sua função primordial em mapear o comportamento dos parâmetros $l$ e $\beta$, através de equações diferenciais de segunda ordem que são função das reações das forças aplicadas nas suspensões.

$$
\begin{gathered}
\ddot{l} . m_{l}+\dot{i} . c_{l}+l . k_{l}=F_{l} \\
\ddot{l}_{r} \cdot m_{\beta}+\dot{l}_{r} \cdot c_{\beta}+l_{r} \cdot k_{\beta}=F_{\beta}
\end{gathered}
$$

Onde $\mathrm{m}$ é a massa suspensa, $\mathrm{c}$ o coeficiente dinâmico e $\mathrm{k}$ o coeficiente estático, $\mathrm{F}$ é a força aplicada na suspensão e $l_{r}$ é a altura do centro da roda traseira. A dependência entre $l_{r}$ e $\beta$, é dada por:

$$
l_{r}=s \cdot \sin \beta
$$

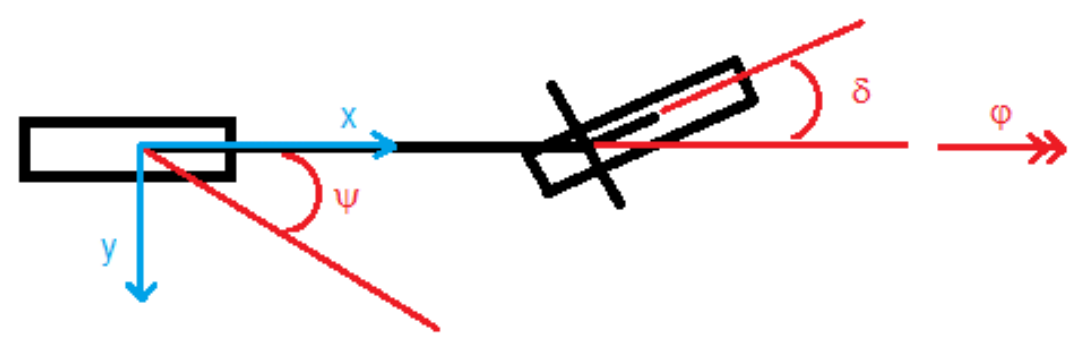

Figura 2 - Geometria da motocicleta (visão superior)

\subsection{Modelo de pneu}

Para o desenvolvimento deste trabalho, o modelo de pneu adotado foi a "Magic Formula", conforme descrito em [3]. Sua forma é descrita por:

$F=D \sin \left[C \tan ^{-1}\left\{B x-E\left(B x-\tan ^{-1} B x\right)\right\}\right]$

Onde $\mathrm{F}$ é a força de reação do pneu, $\mathrm{x}$ é o ângulo de escorregamento, $\mathrm{B}$ o fator de rigidez, C o fator de forma, D reação máxima e $\mathrm{E}$ o fator de curvatura.

\subsubsection{Efeito de forma do pneu}


A inclinação da motocicleta implica na alteração da distância entre o centro da roda traseira e o ponto de contato entre o pneu, aproximado por um toro, e a pista. Esse efeito é exemplificado na figura 3.

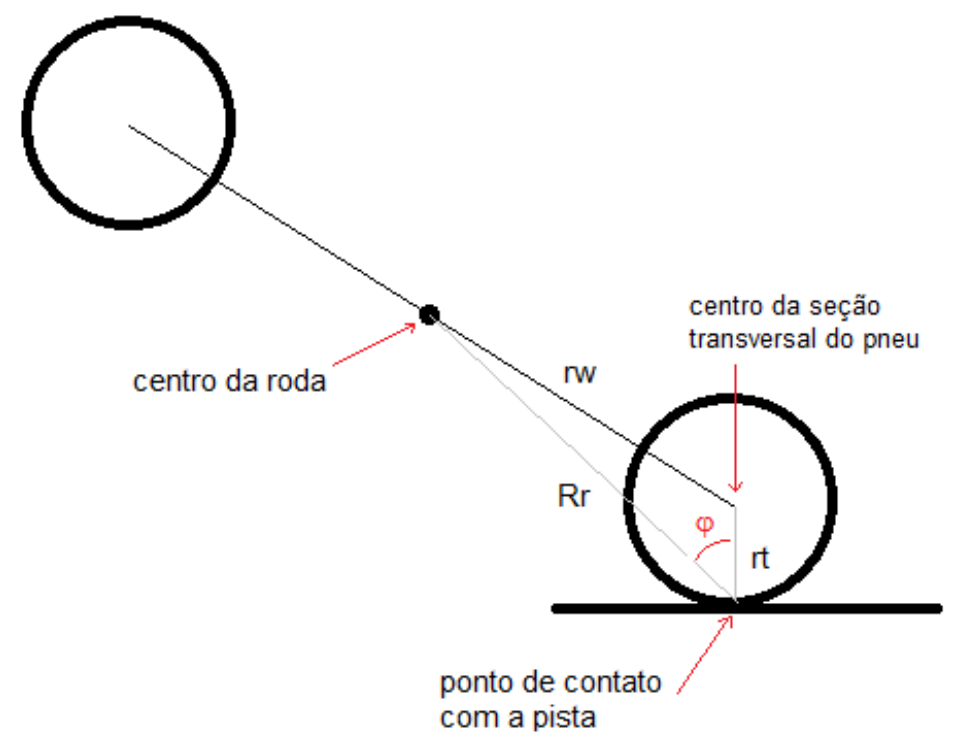

Figura 3 - Raio efetivo da roda traseira com a moto inclinada

Com essa suposição, o raio efetivo, representado por $\mathrm{Rr}$, é dado por:

$$
R_{r}=r_{t} \cdot \cos \varphi+r_{w} \cdot \cos \left(\sin ^{-1}\left(\frac{r_{t}}{r_{w}} \cdot \sin \varphi\right)\right)
$$

Onde, $r_{w}$ é o raio da roda e $r_{t}$ o raio da seção transversal do pneu.

Com isso, pode-se calcular a velocidade longitudinal do veículo como função do raio efetivo e do torque do motor aplicado ao centro da roda. Nesse caso, pode-se simplificar a expressão da velocidade, tomando a velocidade de rotação do motor, representada por $\dot{\theta}$, multiplicada pela relação de marcha, representada por gr, e multiplicando-se novamente pelo raio efetivo. A expressão da velocidade passa então a ser escrita como:

$$
v=\dot{\theta} \cdot g r \cdot R_{r}
$$

\subsection{Dinâmica}

Para direcionar a motocicleta pela pista, é necessário que o piloto aplique torque ao guidão. $O$ esterço do guidão induz a inclinação da motocicleta, que quando está em movimento, é compensada parcialmente pelo efeito giroscópio. O piloto pode influir diretamente na inclinação, aplicando forças nos pedais. Por fim, há também a energia de rotação no movimento das suspensões. 
A energia cinética da motocicleta no deslocamento pode então ser expressa por:

$$
K_{x y}=\frac{1}{2} \cdot m \cdot\left(\dot{x}^{2}+\dot{y}^{2}\right)
$$

Porém, durante o deslocamento, a motocicleta sofre rotações nos eixos longitudinal e vertical e a energia cinética de rotação e inclinação da motocicleta é calculada como:

$$
K_{\varphi \psi}=\frac{1}{2} \cdot m \cdot\left(\begin{array}{ll}
\dot{\varphi} & \dot{\psi}
\end{array}\right) \cdot\left[\begin{array}{cc}
h^{2} & h b \\
b h & b^{2}
\end{array}\right] \cdot\left(\begin{array}{l}
\dot{\varphi} \\
\dot{\psi}
\end{array}\right)
$$

Onde $\mathrm{h}$ é a distância entre a pista e o centro da roda traseira e b é a distância entre $a$ projeção do centro da roda traseira sobre a pista e a projeção do centro de massa da motocicleta sobre a pista. Estas medidas podem ser visualizadas na figura 4.

As distâncias $\mathrm{h}$ e b podem ser descritas como:

$$
\begin{aligned}
& h=d \cdot \cos \left(\gamma-\left(\gamma_{0}-\alpha\right)\right) \\
& b=d \cdot \sin \left(\gamma-\left(\gamma_{0}-\alpha\right)\right)
\end{aligned}
$$

Onde $\gamma_{0}$ é o ângulo de cáster com a motocicleta completamente descarregada.

Deve-se exprimir também as energias cinéticas de rotação das rodas dianteiras e traseiras:

$$
\begin{aligned}
& K_{\theta_{f}}=\frac{1}{4} \cdot m_{f} \cdot r_{f}{ }^{2} \cdot \dot{\theta}_{f}{ }^{2} \\
& K_{\theta_{r}}=\frac{1}{4} \cdot m_{r} \cdot r_{r}{ }^{2} \cdot \dot{\theta}_{r}{ }^{2}
\end{aligned}
$$

Onde $m_{f}$ é a massa da roda dianteira, $r_{f}$ é o raio da roda dianteira, $\dot{\theta}_{f}$ é a velocidade angular da roda dianteira, $m_{r}$ é a massa da roda traseira, $r_{r}$ é o raio da roda traseira e $\dot{\theta}_{r}$ é a velocidade angular da roda traseira.

Pode-se então calcular a energia cinética de rotação do motor: 


$$
K_{\theta}=\frac{1}{4} \cdot m_{m} \cdot r_{m}^{2} \cdot \dot{\theta}^{2}
$$

Onde $m_{m}$ é a massa do motor, $r_{m}$ é o raio das partes rotativas do motor e $\dot{\theta}$ é a velocidade angular do motor.

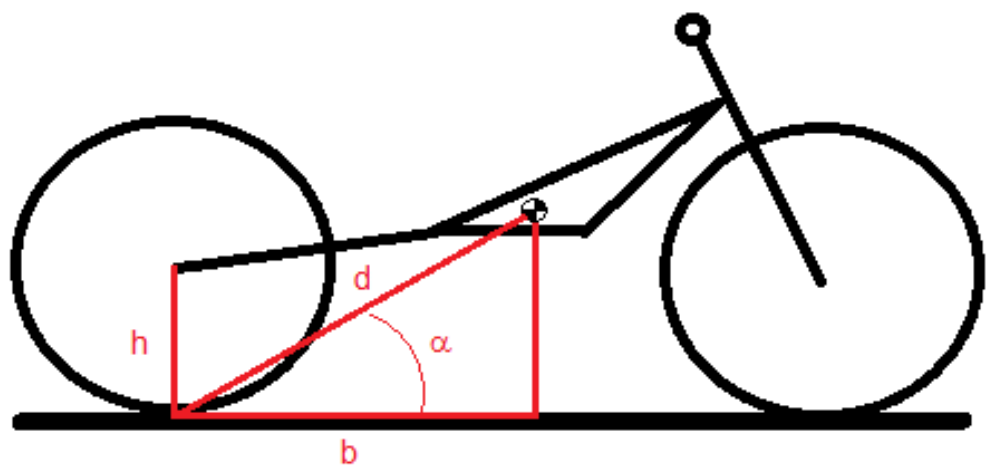

Figura 4 - Coordenadas do centro de massa

A energia cinética total do sistema é dada por:

$$
K=K_{x y}+K_{\varphi \psi}+K_{\theta_{f}}+K_{\theta_{r}}+K_{\theta}
$$

Calculada a energia cinética, o sistema dinâmico é obtido através do cálculo do seu Lagrangeano, que pode ser calculado da seguinte maneira:

$$
\begin{gathered}
\frac{d}{d t} \frac{\partial K}{\partial \dot{\varphi}}-\frac{\partial K}{\partial \varphi}=\Gamma_{\varphi} \\
\frac{d}{d t} \frac{\partial K}{\partial \dot{\theta}}-\frac{\partial K}{\partial \theta}=\Gamma_{\theta}
\end{gathered}
$$

O sistema pode então ser escrito em forma matricial:

$$
M .\left[\begin{array}{l}
\ddot{\varphi} \\
\ddot{\theta}
\end{array}\right]+V=\tau
$$

Onde,

$$
\tau=\left[\begin{array}{l}
\Gamma_{\varphi} \\
\Gamma_{\theta}
\end{array}\right]
$$




$$
\begin{gathered}
M=\left[\begin{array}{cc}
\frac{\partial^{2} K}{\partial \dot{\varphi}^{2}} & \frac{\partial^{2} K}{\partial \dot{\varphi} \partial \dot{\theta}} \\
\frac{\partial^{2} K}{\partial \dot{\theta} \partial \dot{\varphi}} & \frac{\partial^{2} K}{\partial \dot{\theta}^{2}}
\end{array}\right] \\
V=\left[\begin{array}{cc}
-\frac{\partial K}{\partial \varphi}+\frac{\partial^{2} K}{\partial \dot{\varphi} \partial \varphi} \cdot \dot{\varphi}+\frac{\partial^{2} K}{\partial \dot{\varphi} \partial \theta} \cdot \dot{\theta} \\
-\frac{\partial K}{\partial \varphi}+\frac{\partial^{2} K}{\partial \dot{\theta} \partial \varphi} \cdot \dot{\varphi}+\frac{\partial^{2} K}{\partial \dot{\theta} \partial \theta} \cdot \dot{\theta}
\end{array}\right]
\end{gathered}
$$

É necessário acrescentar a reação normal à força peso do veículo, bem como o efeito da força centrípeta ao descrever movimentos não retilíneos. Essas forças são aplicadas no centro de massa e são incorporadas ao modelo como torques externos ao sistema $\tau_{\text {ext }}$.

Então, o sistema completo passa a ser descrito como:

$$
\left[\begin{array}{cc}
\frac{\partial^{2} K}{\partial \dot{\varphi}^{2}} & \frac{\partial^{2} K}{\partial \dot{\varphi} \partial \dot{\theta}} \\
\frac{\partial^{2} K}{\partial \dot{\theta} \partial \dot{\varphi}} & \frac{\partial^{2} K}{\partial \dot{\theta}^{2}}
\end{array}\right]\left[\begin{array}{c}
\ddot{\varphi} \\
\ddot{\theta}
\end{array}\right]+\left[\begin{array}{c}
-\frac{\partial K}{\partial \varphi}+\frac{\partial^{2} K}{\partial \dot{\varphi} \partial \varphi} \cdot \dot{\varphi}+\frac{\partial^{2} K}{\partial \dot{\varphi} \partial \theta} \cdot \dot{\theta} \\
-\frac{\partial K}{\partial \varphi}+\frac{\partial^{2} K}{\partial \dot{\theta} \partial \varphi} \cdot \dot{\varphi}+\frac{\partial^{2} K}{\partial \dot{\theta} \partial \theta} \cdot \dot{\theta}
\end{array}\right]=\left[\begin{array}{c}
\Gamma_{\varphi} \\
\Gamma_{\theta}
\end{array}\right]+\left[\begin{array}{c}
N \cdot h \cdot \sin \varphi-T \cdot h \cdot \cos \varphi \\
0
\end{array}\right]
$$

\subsubsection{Modelo de pista}

Para a validação do modelo apresentado até aqui, o sistema dinâmico descrito pela equação 19 necessita do desenho de um controle, que faz as vezes de piloto da motocicleta, inserindo torque no guidão, bem como variando a aceleração, e para tanto, o sistema de controle necessita de uma referência da pista. Essa referência pode ser a linha média da largura da pista, com variações restritas aos extremos do asfalto. As pistas testadas aqui serão o autódromo de Interlagos em São Paulo e o circuito Motorland Aragón, em Alcañiz, na Espanha.

\section{Controle ótimo}

O problema de controle ótimo de processo é composto por um modelo matemático que represente a planta a ser controlada, como o modelo expresso na equação 19, a descrição das restrições físicas, neste caso, a largura da pista, as condições de contorno, como o ângulo de inclinação, que em uma motocicleta esportiva fica entre $60^{\circ}$ e $+60^{\circ}$ e um critério de performance, como o tempo de volta mínimo.

O sistema dinâmico é expresso como um sistema de equações diferenciais ordinárias de primeira ordem com a seguinte forma: 
$\dot{x}(t)=a(x(t), u(t), t), \operatorname{com} x\left(t_{0}\right)=x_{0}$, e $t \in\left[t_{0}, t_{f}\right]$

Onde x é o conjunto de variáveis de estado do sistema e u é o conjunto de sinais aplicado ao sistema.

O papel das restrições físicas é garantir que as variáveis de estado e de controle do sistema assumam valores que façam sentido para o problema analisado. No caso da minimização do tempo de volta, o máximo torque de aceleração e frenagem limitam o movimento longitudinal, enquanto que o movimento transversal é limitado pelo ângulo de esterço. Ao final, as coordenadas da projeção do centro de massa sobre a pista, devem permanecer dentro dos limites do asfalto. As restrições são expressas como:

$$
c(t)=c(x(t), u(t), t) \leq 0, \mathrm{t} \in\left[t_{0}, t_{f}\right]
$$

De maneira similar, as condições de contorno têm a forma:

$$
u_{L} \leq u(t) \leq u_{U}, \mathrm{t} \in\left[t_{0}, t_{f}\right]
$$

O conjunto sequencial de sinais que satisfazem as condições de contorno é chamado de histórico de controles admissíveis, e ao mesmo tempo, a trajetória dos estados que respeita as restrições é uma trajetória realizável.

Para completar o problema, é necessário definir o critério de performance, que descreve uma função dos estados finais do sistema, assim como o funcional das trajetórias de estado e do histórico de controles. Aqui o funcional pode ser entendido como uma função de funções e o critério de performance pode ser descrito como:

$$
J=\int_{t_{0}}^{t_{f}} f(x(t), u(t), t) d t
$$

A solução do problema de controle ótimo de processo visa encontrar um controle admissível $u^{*}$, que provoque o sistema a descrever uma trajetória realizável $x^{*}$, que minimiza o critério de desempenho $J$. 


\subsection{Função de performance}

A função $f$ exposta em 23 pode assumir duas formas: com o tempo $t_{f}$ livre ou determinado. A opção de se ter $t_{f}$ livre é a mais intuitiva, porém, implicaria em adotar termos adicionais à equação 23. Neste trabalho, adotou-se $t_{f}$ fixo, ou seja, o problema a ser resolvido é simular uma motocicleta se deslocando longitudinalmente o mais rápido possível dentro de um dado intervalo de tempo.

Com esse objetivo, adotou-se a função de performance abaixo, que foi adaptada de [4]:

$f=-\frac{v}{v_{0}}+\left(\frac{s_{2}}{l_{\text {pista }} / 2}\right)^{2 n}+\left(\frac{F_{y r}{ }^{2}+F_{x r}{ }^{2}}{\left(\mu m g \frac{(1-d \cos \alpha)}{w_{i}}\right)^{2}}\right)^{n}+\left(\frac{F_{y f}{ }^{2}+F_{x f}{ }^{2}}{\left(\mu m g \frac{(d \cos \alpha)}{w_{i}}\right)^{2}}\right)^{n}+\left(\frac{d \dot{\psi}}{d t}\right)^{2}+\left(\frac{d \dot{\varphi}}{d t}\right)^{2}$

O termo mais importante é o primeiro, que é o responsável por maximizar a velocidade v. O valor de $v_{0}$ na realidade é arbitrário, e visa tornar o termo adimensional. Todos os termos foram calculados de forma a terem valores positivos e inferiores a um, ao ultrapassar esse valor, a integral de 23 começa a tomar valores cada vez maiores, penalizando o algoritmo de minimização.

O segundo termo é responsável por manter a motocicleta dentro dos limites da pista, e sendo assim, a variável $s_{2}$ representa a distância da posição da motocicleta ao centro da pista, cuja distância máxima é ${ }^{l_{\text {pista }} / 2}$, sempre que essa distância aumentar, esse termo também penaliza a minimização. Assim, tem-se uma relação onde o distanciamento do centro da pista, é compensado por maiores velocidades.

O terceiro e quarto termos determinam as condições de aderência dos pneus, com $F_{y r}$ e $F_{x r}$ sendo as forças exercidas pelo pneu traseiro no solo na direção lateral e longitudinal respectivamente, $F_{y f}$ e $F_{x f}$, são seus correspondentes no pneu dianteiro e $\mu$ o coeficiente de atrito entre os pneus e o solo.

Os demais termos são colocados de forma a reduzir a incidência de mudanças bruscas nos valores dos controladores.

O expoente $n$ tem a função de enfatizar os efeitos, sua ausência implicaria na predominância do primeiro termo e a motocicleta aceleraria indefinidamente até o limite de rotações do motor, sempre em linha reta, sem respeitar as condições de aderência ideais para uma corrida. 
Todas as variáveis de $\mathrm{f}$, quando substituídas por seus respectivos valores, tornam $\mathrm{o}$ critério de performance uma função das variáveis de controle $\delta, \Gamma_{\varphi}$ e $\Gamma_{\dot{\theta}}$, que são submetidos à otimização.

\section{Resultados}

\section{Circuito de Interlagos}

O próximo passo foi utilizar a metodologia desenvolvida até aqui, aplicando-a ao traçado do circuito de Interlagos.

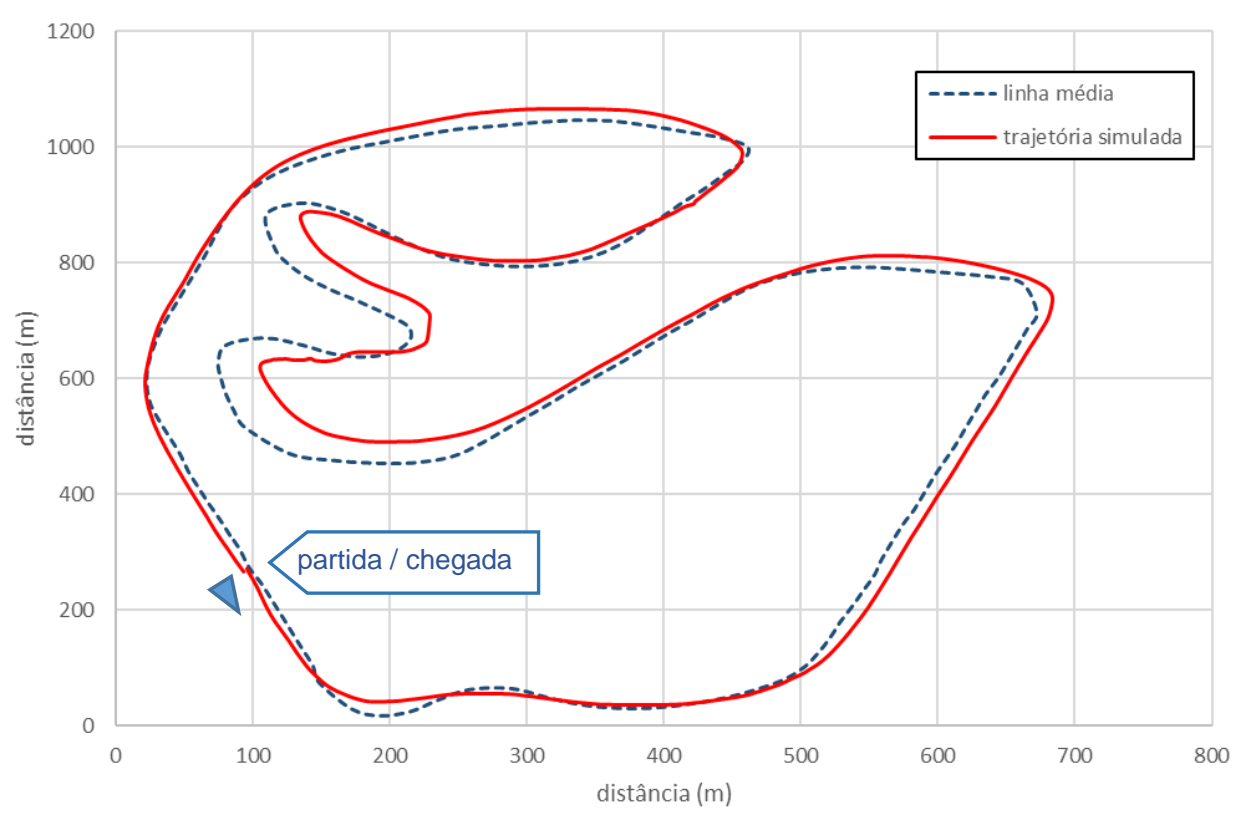

Figura 5 - Trajetória simulada comparada com a linha média da pista de Interlagos.

De forma geral, o modelo respondeu conforme o esperado, abrindo a trajetória nas tomadas das curvas, seguindo depois uma curvatura cada vez menor até o ponto de tangencia e abrindo o trajeto na saída da curva, conforme a velocidade aumenta. Porém, a figura 5 deixa bastante claro também que a trajetória nem sempre respeitou o limite da pista, e esse comportamento pode ser atribuído aos seguintes fatores:

- Comportamento dos dados da pista de referência: Os dados devem ser diferenciáveis em todos os pontos. No caso dos dados de Interlagos, o modelo respondeu muito bem quando os dados de referência eram bem comportados, seja em retas ou curvas, entretanto, alterações abruptas da trajetória, mesmo que pequenas, fazem que o modelo perca a referência, obrigando que a simulação seja reiniciada nesse ponto, com intervenção no ângulo $\psi_{0}$ para que a simulação continue. 
- Resposta do conjunto motriz: na figura 7 é mostrada a curva da velocidade, que é função do acionamento do acelerador, demonstrado na figura 6 . As velocidades demonstradas no início da volta são factíveis, dado que a motocicleta inicia seu percurso com velocidade de $255 \mathrm{~km} / \mathrm{h}$, porém, ainda que as condições de aderência sejam respeitadas, a velocidade de tomada da curva após o final da reta principal é bastante elevada. Esse efeito pode ser atribuído ao conjunto motriz simplificado, onde a aceleração angular do motor varia livremente e não há perdas por troca de marcha. A velocidade é compensada pela inclinação, que atingiu o valor limite diversas vezes durante o contorno das primeiras curvas.
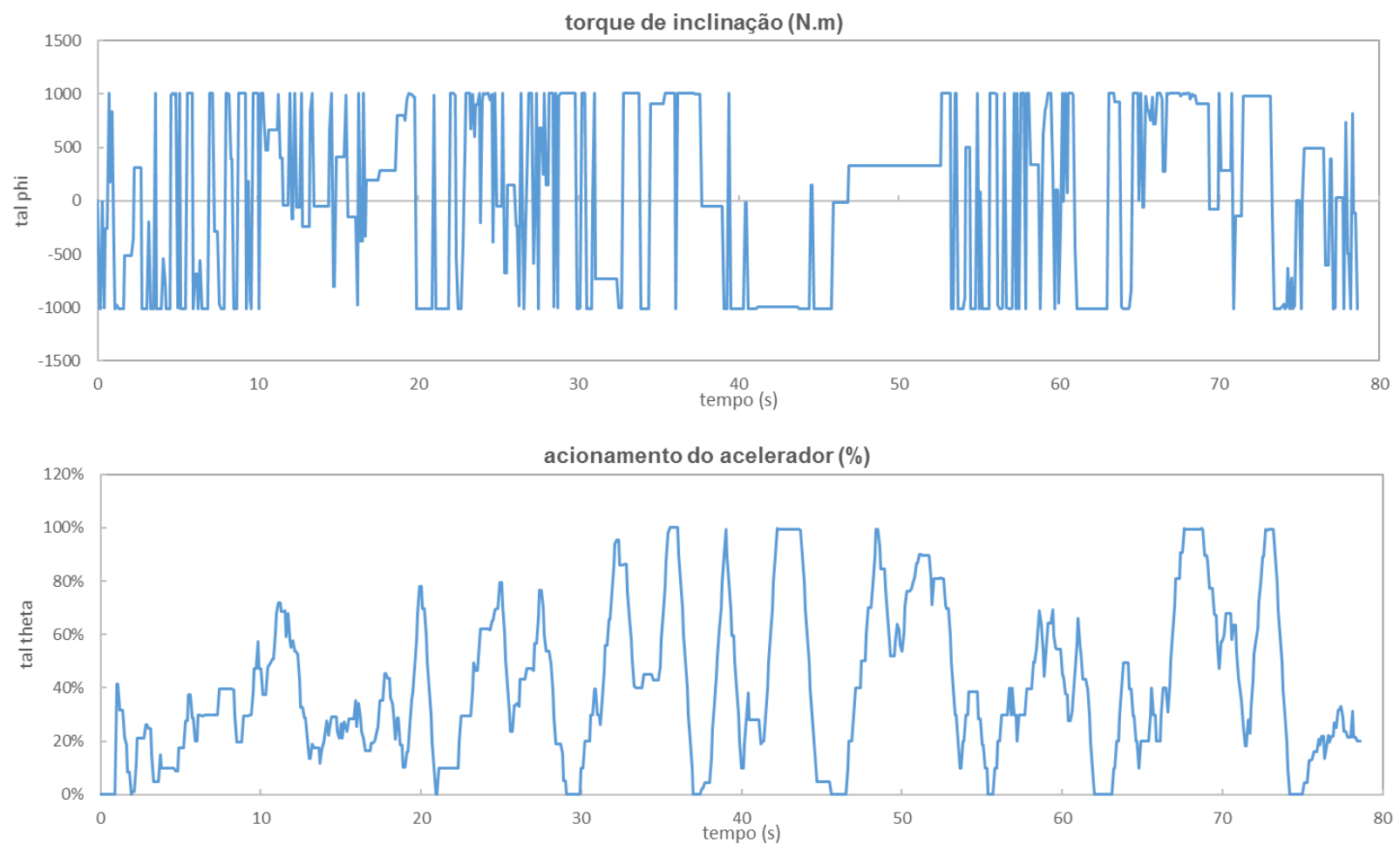

Figura 6 - Comportamento simulado da motocicleta em Interlagos.

- Ausência da terceira dimensão. Interlagos é um circuito conhecido pelos pilotos por explorar bastante o relevo do terreno em seu traçado, a curva "S do Senna" é uma descida acentuada, enquanto que a "curva da junção", é um aclive que demanda bastante dos motores. Esses efeitos não foram considerados na modelagem da pista.

O tempo da volta foi estimado em 1 min e $19 \mathrm{~s}$.

No histórico dos controles simulados, os valores máximos e mínimos apresentados são valores viáveis para a condução da motocicleta, porém, a velocidade em que variam parece demasiadamente elevada. A alta frequência dos controles indica uma oportunidade de desenvolvimento do modelo, por ser necessário controlar esse comportamento. 

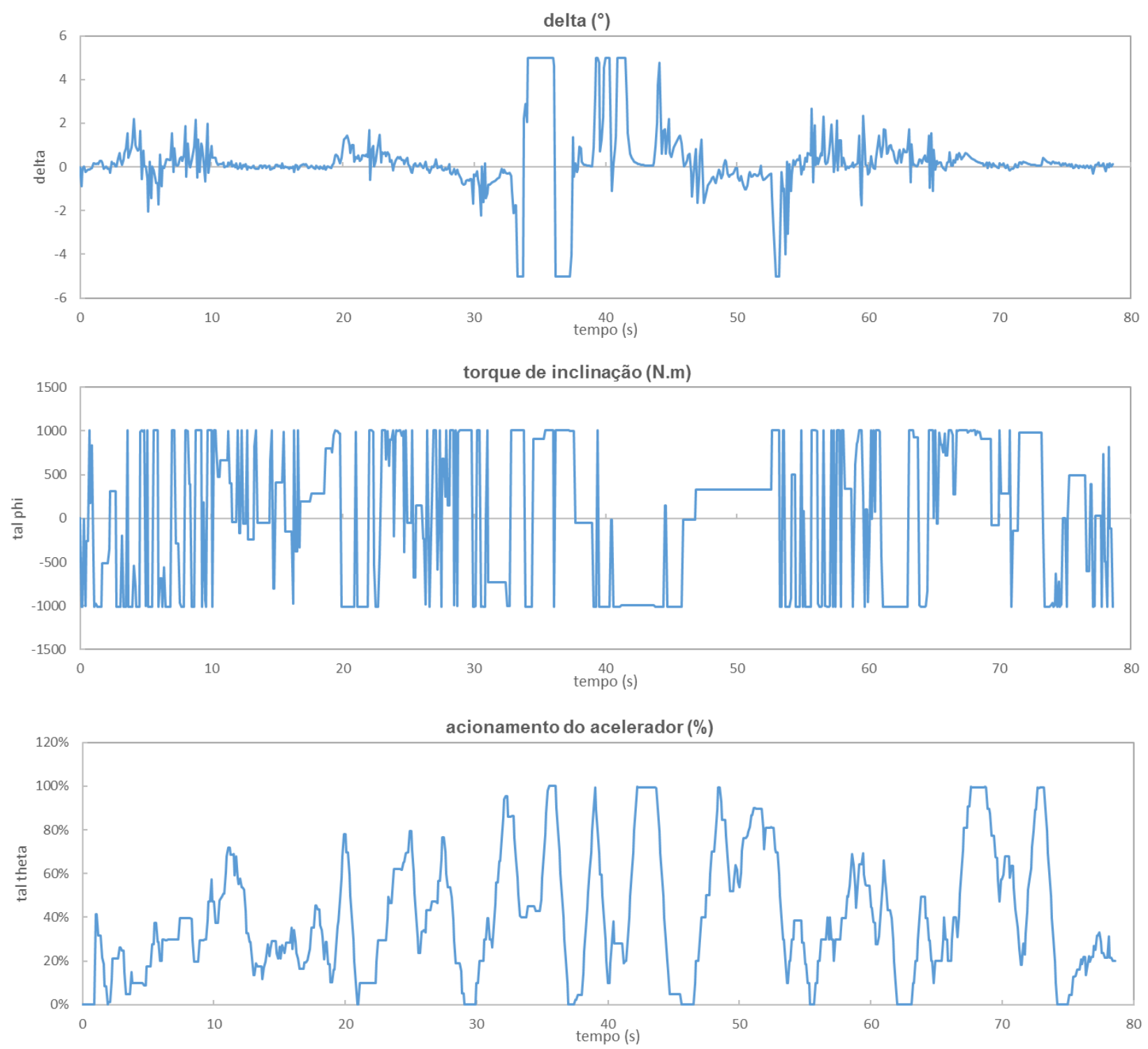

Figura 1 - Histórico dos controles simulados na pista de Interlagos.

\begin{tabular}{|c|c|c|c|}
\cline { 2 - 4 } \multicolumn{1}{c|}{} & pista & simulação & real \\
\hline distância percorrida $(\mathrm{m})$ & 4309 & 4149 & 4391 \\
\hline tempo de volta & - & $1 \mathrm{~min} 19 \mathrm{~s}$ & $1 \mathrm{~min} 46 \mathrm{~s}$ \\
\hline velocidade média $(\mathrm{km} / \mathrm{h})$ & - & 190,03 & 149,79 \\
\hline tempo em aceleração $(\mathrm{s})$ & - & 19,8 & - \\
\hline tempo em frenagem $(\mathrm{s})$ & - & 59,2 & - \\
\hline
\end{tabular}

Tabela 1 - Resumo da simulação de Interlagos

Como referência de comportamento, foram obtidos dados reais para o circuito de Interlagos, onde uma motocicleta Ducati 1198 foi pilotada por um piloto amador. A 
captura dos dados foi através do sistema incluso na própria motocicleta. Este sistema é chamado de Ducati DDA, e é fabricado por [5]. O sistema coleta várias informações da central eletrônica da motocicleta e os armazena em um dispositivo de memória para posterior consulta.
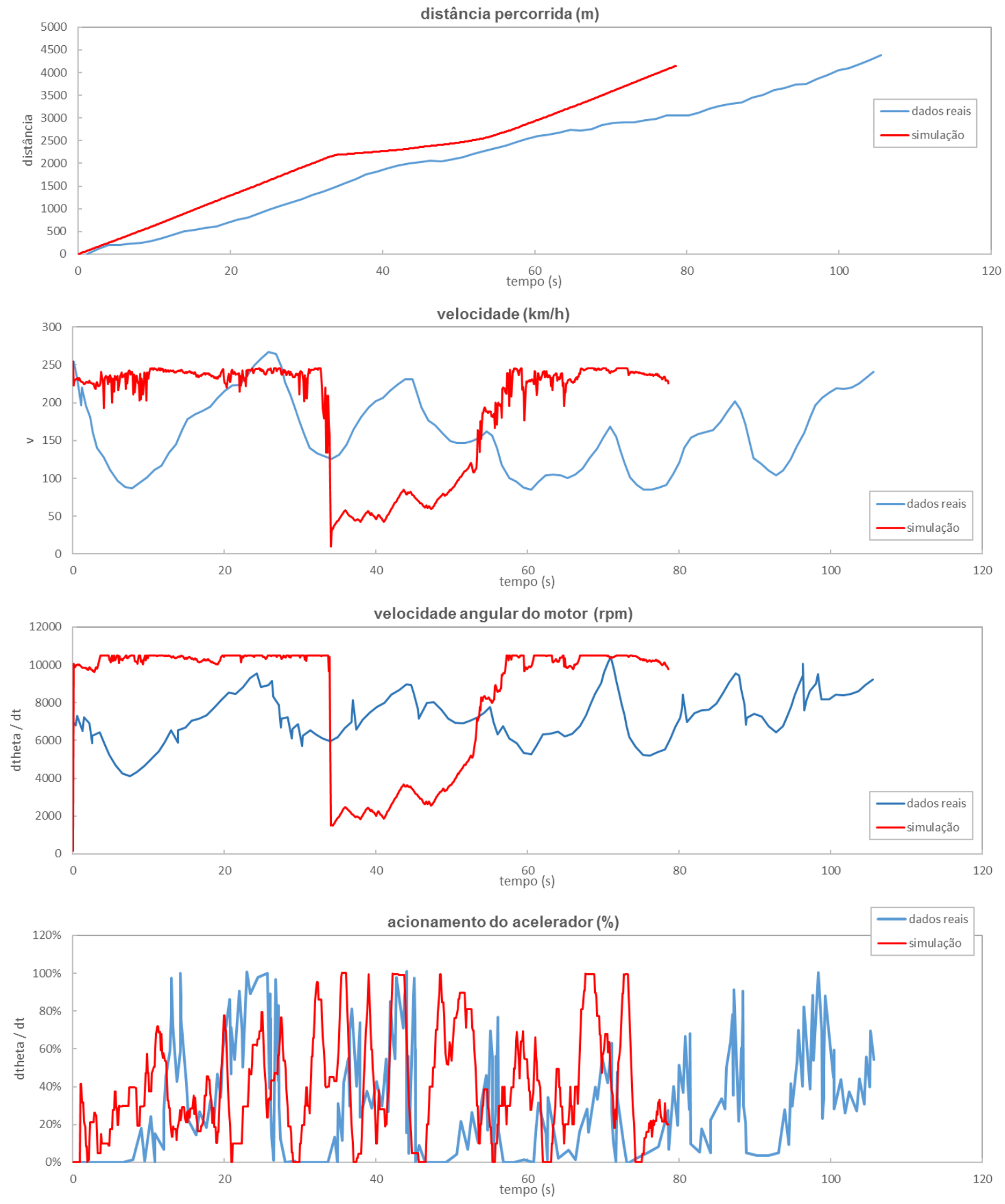

Figura 8 - Comparação entre os resultados da simulação e os obtidos em condições reais em Interlagos. 
Com os dados reais, observa-se que a volta simulada é completada em um tempo bastante inferior à volta real, 1 min e $19 \mathrm{~s} \mathrm{e} 1$ min e $46 \mathrm{~s}$ respectivamente. Boa parte da diferença de tempos, entretanto, não se dá apenas pelas imprecisões do modelo, mas também por outra premissa ainda não considerada, que é a presença de um piloto humano, sujeito a indecisões, fatiga e receios.

$\mathrm{Na}$ estrutura construída no modelo, assume-se que as condições de aceleração e frenagem, bem como o limite de aderência, sempre são levados ao limite, resultando no comportamento ótimo. Ainda que tivéssemos um piloto profissional conduzindo a motocicleta neste circuito, dificilmente um ser humano seria capaz de pilotar por toda a volta explorando o máximo do equipamento, ou seja, os fatores emocionais e a exaustão física do piloto são itens importantes no desempenho real de um veículo de competição, e esses fatores não foram sequer levados em consideração nas simulações.

As principais medidas da volta foram resumidas na tabela 1.

Os resultados mostrados na figura 8 ilustram bem a simplificação do conjunto motriz. A velocidade angular do motor mantém-se a mesma a maior parte do tempo, obrigando a velocidade a manter-se também estável. Ainda que os valores estejam longe do ideal, a correlação entre a rotação do motor e a velocidade é uma característica intuitiva e esperada, e pode ser observada também nos dados reais

\section{Circuito Motorland Aragón}

Neste item, será demonstrado o resultado da simulação aplicada ao circuito de Aragón. A crítica aos resultados é similar ao que foi discutido na simulação de Interlagos, porém, neste caso, o circuito foi dividido em vários setores independentes e seus traçados foram aproximados por curvas paramétricas, com isso, foi possível aumentar consideravelmente a resolução dos dados, o que se mostrou positivo no que tange a manter a motocicleta dentro dos limites da pista. Esse tratamento também implicou a eliminação da grande parte dos pontos não diferenciáveis presentes nos dados brutos. 


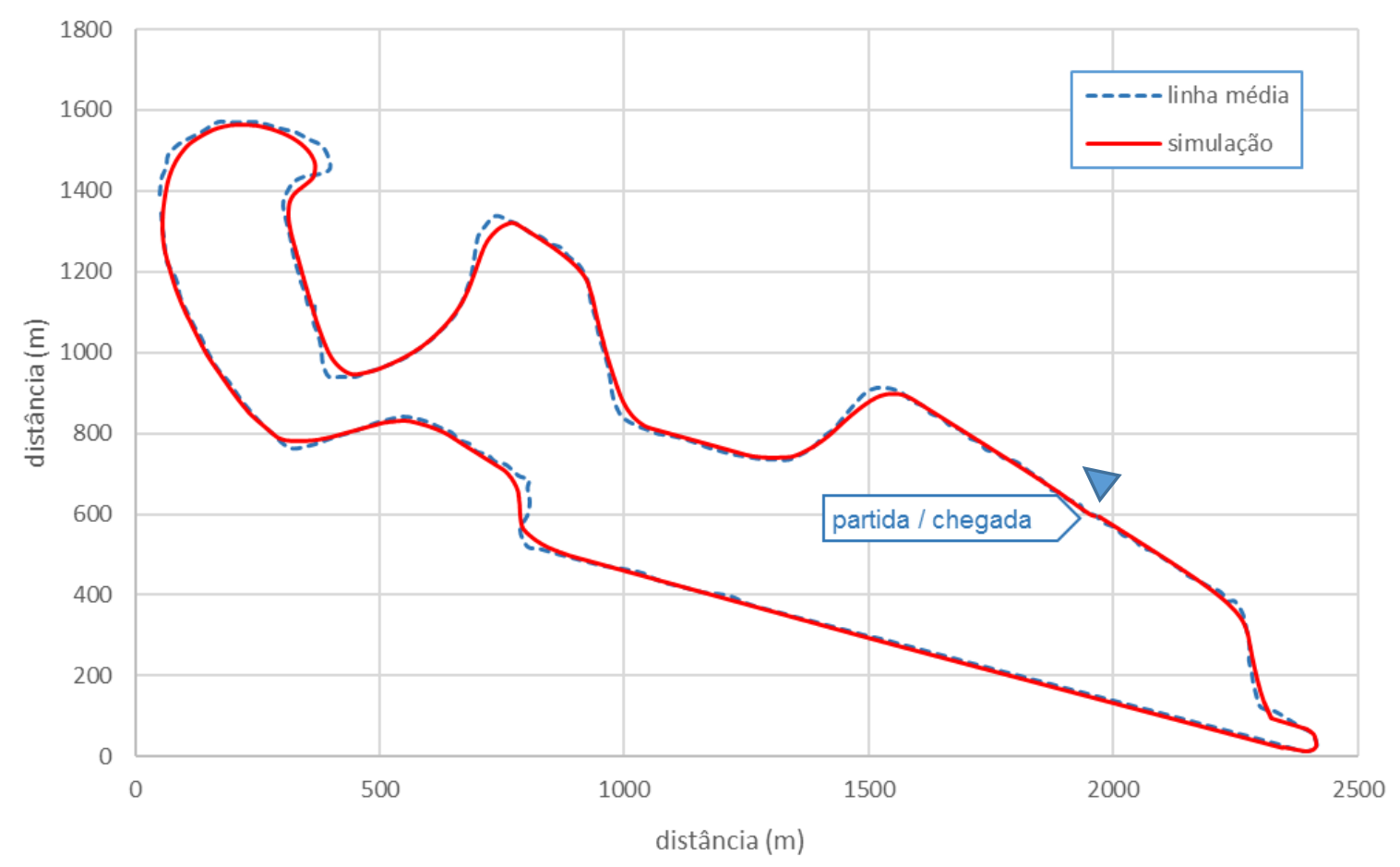

Figura 9 - Trajetória simulada comparada com a linha média da pista de Aragón.

No início da simulação, há uma queda da velocidade inicial, que pode ser atribuída ao descasamento dos valores iniciais da simulação e a relação entre o giro do motor e a velocidade produzida no modelo. Porém, o trajeto simulado permitiu que a velocidade se mantivesse a mais elevada possível, caindo nas entradas de curva. Esse efeito é especialmente observado na curva ao final da reta oposta.

Na simulação também se destaca o histórico de ângulos de inclinação, que apresenta valores máximos e mínimos similares ao que é visto em uma condução profissional.

\begin{tabular}{|c|c|c|c|}
\cline { 2 - 4 } \multicolumn{1}{c|}{} & pista & simulação & real \\
\hline distância percorrida $(\mathrm{m})$ & 5344 & 5207 & 5428 \\
\hline tempo de volta & - & $1 \min 56 \mathrm{~s}$ & $1 \mathrm{~min} 58 \mathrm{~s}$ \\
\hline velocidade média $(\mathrm{km} / \mathrm{h})$ & - & 162,16 & 165,81 \\
\hline tempo em aceleração $(\mathrm{s})$ & - & 19,8 & - \\
\hline tempo em frenagem $(\mathrm{s})$ & - & 59,2 & - \\
\hline
\end{tabular}

Tabela 2 -Resumo da simulação de Aragón

De forma geral, o histórico de controles é coerente com os efeitos gerados pela motocicleta na simulação, os ângulos de esterço são relativamente pequenos e há 
forte atuação do torque de inclinação, o que destaca a intensa movimentação do piloto sobre a motocicleta em uma condução desse tipo.

Apesar do desempenho ter melhorado bastante, ainda assim, o modelo não respeitou os limites da pista em todo o trajeto, principalmente nas curvas mais acentuadas. Entretanto, novamente nota-se tangenciamento das curvas, produzindo um trajeto que favorece à motocicleta ter velocidades mais elevadas. A trajetória simulada está na figura 9.
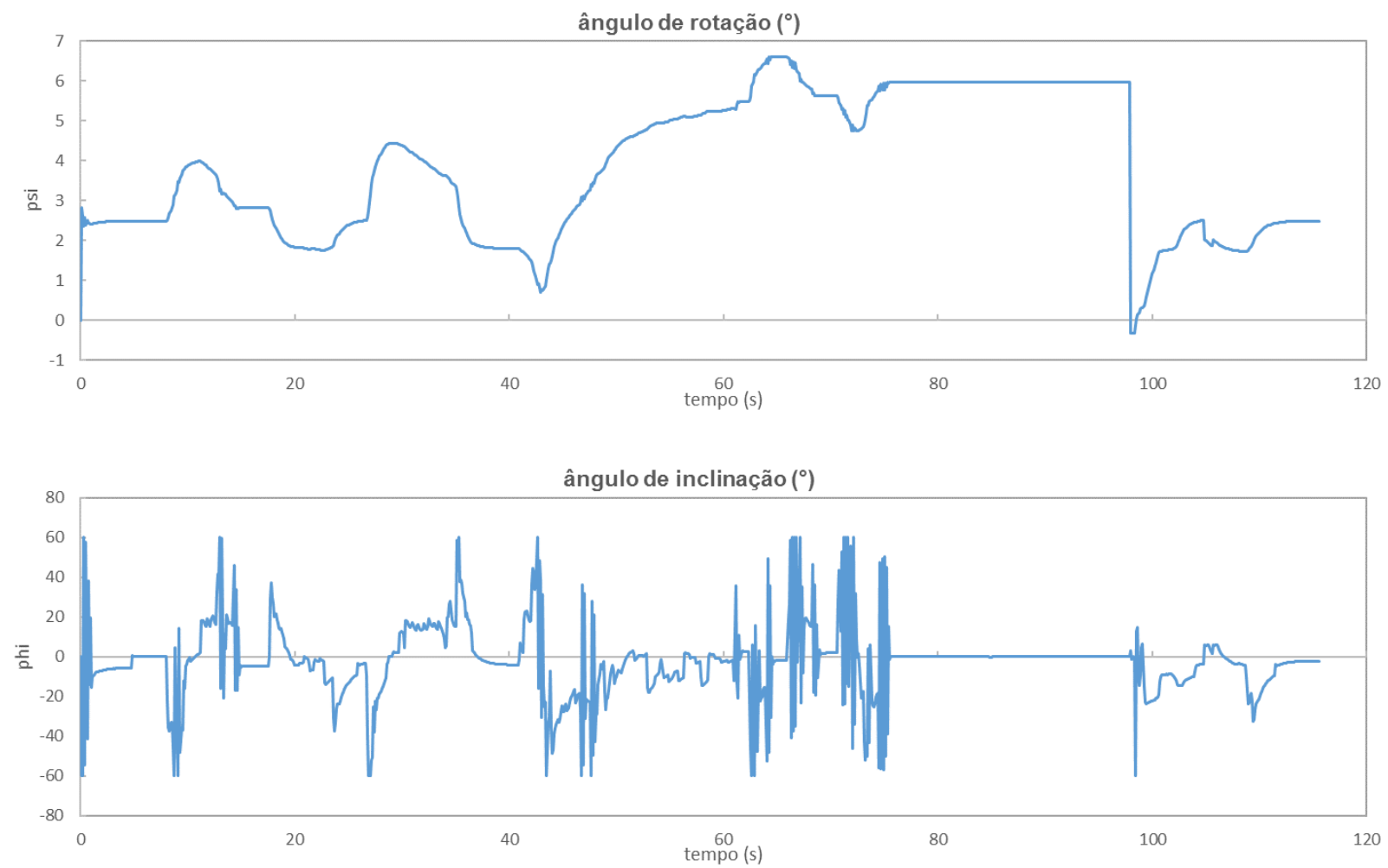

Figura 2 - Comportamento simulado da motocicleta em Aragón.

Por volta dos $85 \mathrm{~s}$ de simulação, pode-se observar a tomada da curva ao final da reta oposta, a aceleração cessa na frenagem, mas durante todo o contorno da curva, entre os tempos 85 e 95 s há um pequeno acionamento do acelerador, característica bastante peculiar, pois para o piloto essa pequena aceleração estabiliza a motocicleta na curva, facilitando a condução.

Os resultados da simulação em Aragón foram melhores que os observados na simulação anterior, essencialmente por conta dos dados de referência da pista terem qualidade bastante superior. Como pode ser visto na tabela 2, os tempos de volta simulado e real foram relativamente próximos, assim como a velocidade média observada. 
Para avaliação dos resultados, foram utilizados dados reais capturados de uma volta de um piloto profissional, conduzindo uma motocicleta de competição equipada com o sistema WINTAX4, fabricado por [6], que é um sistema profissional de coleta de dados de veículos de competição.
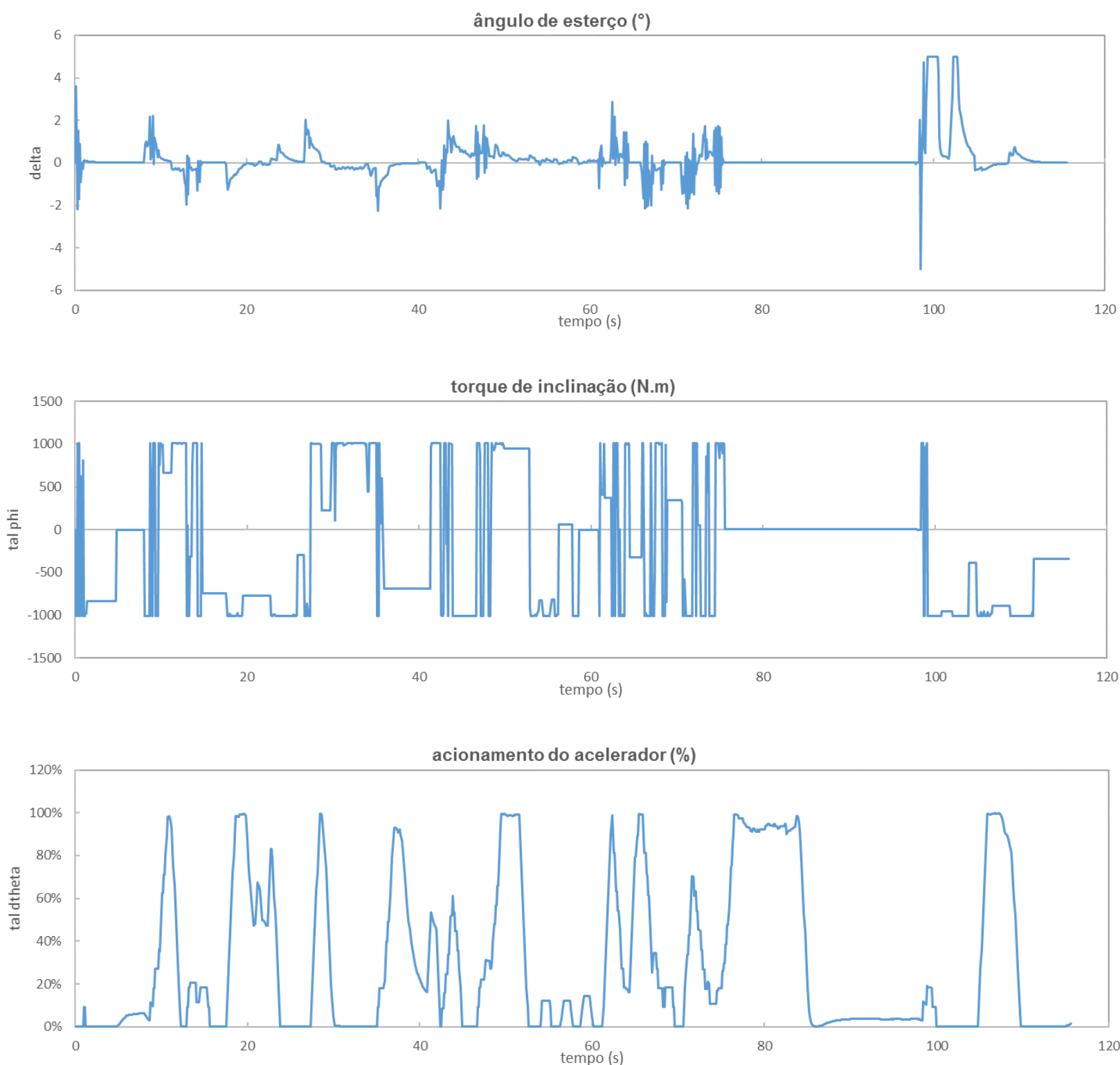

Figura 3 - Histórico dos controles admissíveis simulados na pista de Aragón.

O primeiro gráfico da figura 12 mostra uma aderência relevante da evolução a distância percorrida no tempo entre os dados obtidos pelo modelo e a informação real. Mesmo a velocidade simulada guarda melhor relação com os dados reais, com destaque para o final da volta, onde por volta dos $90 \mathrm{~s}$, há uma forte frenagem para a tomada da curva final. 


\section{CONCLUSÃO}

O modelo desenvolvido serve de ponto de partida para o entendimento mais profundo da engenharia aplicada ao esporte a motor. Além das simulações apresentadas, poder-se-ia estudar o efeito de mudar a geometria da moto, mudar a altura do centro de gravidade, mudar a distância entre eixos, mudar a aderência dos pneus.
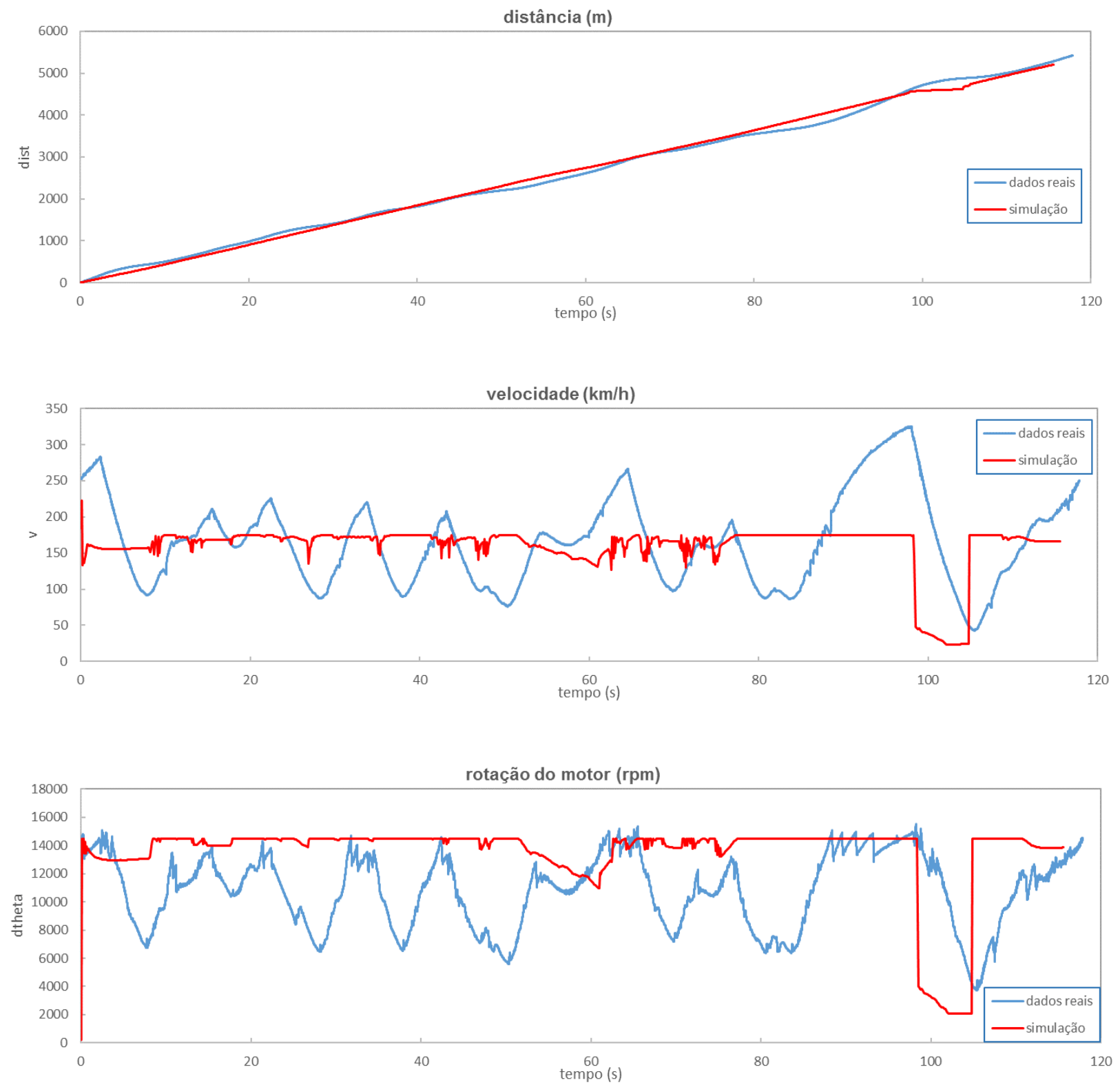

Figura 12 - Comparação entre os resultados da simulação e os obtidos em condições reais em Aragón. 
Cada uma dessas características interfere no desempenho e no manuseio do veículo, e apenas o vislumbre dessas muitas possibilidades de ajuste da motocicleta já representa, por si, um passo ao aprofundamento do tema.

Grande parte do desafio de se construir um modelo como este reside na obtenção dos parâmetros, tanto os dados da pista, quanto a informação obtida de pilotos reais, apresentam pontos de difícil interpretação, que demandam grande interação entre o desenvolvedor do modelo e os fornecedores de informação.

Apesar das discrepâncias observadas, o modelo parece promissor, visto que existem ainda grandes simplificações neste desenvolvimento. Um trabalho mais profundo de calibragem de parâmetros permitiria 0 uso do modelo no propósito de seu desenvolvimento, indicar a melhor trajetória, bem com indicar onde, e quanto, acelerar durante a volta. Os pequenos ângulos de esterço simulado nas trajetórias, bom como a intensa movimentação do piloto no topo da motocicleta já oferecem informações para o piloto iniciante, indicando de forma geral que o esterço na pista deve ser pequeno, e isto só é possível, se houver deslocamento do centro de massa da moto com a adequada movimentação do piloto.

\section{REFERÊNCIAS}

[1] DE VITTORI, Marco. Modeling and Control of a Motorbike. Lisboa, Instituto Superior Técnico da Universidade Técnica de Lisboa, julho de 2011.

[2] CASANOVA, D. On Minimum Time Vehicle Manoeuvring: Theoretical Optimal Lap. Cranfield, Cranfield University, School of Mechanical Engineering, Cranfield, novembro de 2000 ..

[3] Pacejka, H. Tire and Vehicle Dynamics 2nd ed., Burlington, MA, ButtworthHeinemann, 2006.

[4] COSSALTER, V. et al. A General Method for the Evaluationof Vehicle Manoeuvrability with Special Emphasis on Motorcycles. Vehicle System Dynamics: International Journal of Vehicle Mechanics and Mobility.Volume 31, 1999. Disponível em :http://www.tandfonline.com/doi/abs/10.1076/vesd.31.2.113.2094., Acesso em 04 jan 2016.

[5] Prosa.com. Marghera, VE, Itália. Ducati Data Analyzer Web Support. Disponível em: http://dda.prosa.com/. Acesso em: 04 mai 2016.

[6] Magnetti Marelli. WINTAX4 PRO Data acquisition and analysis Telemetry Level. Disponível em: http://www.magnetimarelli.com/sites/default/files/2_SF_Wintax_PRO_rev8.0_1.pdf. Acesso em 04 mai 2016. 